\title{
Percepción de riesgos y consumo de drogas en estudiantes universitarios de enfermería, Arequipa, Perú ${ }^{1}$
}

Fidel Urday-Concha ${ }^{2}$, Caridad Gonzáles-Vera ${ }^{3}$, Lorenzo Jesús Peñalva Suca ${ }^{4}$, Esther Lourdes PantigosoBustamante $^{5}$, Serafín Humberto Cruz Sánchez ${ }^{6}$, Lino Pinto-Oppe ${ }^{7}$

Institución: Universidad Alas Peruanas (UAP) y Universidad Nacional de San Agustín (UNSA)

\section{RESUMEN}

El objetivo del presente estudio fue tener una primera aproximación sobre la prevalencia de vida, información, percepciones, y comportamientos de riesgo respecto del consumo de drogas lícitas, ilícitas y médicas, según sexo, en estudiantes de enfermería. Se realizó un estudio cuantitativo, descriptivo exploratorio y corte transversal, tipo censo. Se aplicó un cuestionario estandarizado de 241 ítems y previamente aplicado a 86 estudiantes del tercer año de enfermería de una universidad particular de Arequipa Metropolitana. Se encontró altas prevalencias de vida para el consumo de sustancias lícitas, $84,4 \%$ en bebidas alcohólicas y $62,8 \%$ en tabaco, y para cualquier droga ilícita $31,4 \%$, entre las que destacan la marihuana $(17,1 \%)$ y cocaína $(4,6 \%)$, con diferencias de consumo a favor de los hombres, mientras que en las drogas médicas la sustancia más usada corresponde a tranquilizantes $(19,8 \%)$, con diferencias de consumo a favor de las mujeres. Asimismo, en la universidad más del $93 \%$ de los estudiantes recibió información para prevenir el uso de drogas, y el 34,8\% declaró haber tenido relaciones sexuales entre una y varias veces bajo los efectos del alcohol y drogas ilícitas. Se concluye que, los resultados evidencian la magnitud de la problemática social del consumo de drogas lícitas, ilícitas y médicas, entre los estudiantes universitarios; además, hay necesidad de desarrollar en la carrera de enfermería estrategias de promoción de la salud y prevención del consumo de sustancias psicoactivas para reducir y evitar que las prevalencias del consumo se incrementen entre los futuros profesionales promotores de estilos de vida saludables, para garantizar una mejor calidad de vida en la población.

Palabras clave: drogas-ilícitas; estudiantes-de-enfermería; percepción-de-riesgos; prevalencia.

DOI: $10.15517 /$ revenf.v0i36.33416

${ }^{1}$ Fecha de recepción: 23 de mayo del 2018

Fecha de aceptación: 4 de octubre del 2018

${ }^{2}$ Sociólogo. Doctor en Sociología y doctorando en Ciencias Biomédicas. Profesor Escuela de Postgrado, Universidad Alas Peruanas (UAP) y Universidad Nacional de San Agustín (UNSA), Arequipa, Perú. Correo electrónico: fidelurdayconcha@hotmail.com

${ }^{3}$ Enfermera. Estudios de Maestría en Ciencias de Enfermería. Profesora Principal a D.E., UNSA. Perú. Correo electrónico: caridadgonzalesvera@hotmail.com

${ }^{4}$ Sociólogo. Doctor en Educación Superior. Profesor Escuela Profesional de Enfermería, UAP. Perú. Correo electrónico: jesusleo55@hotmail.com

${ }^{5}$ Psicóloga. Especialista en farmacodependencia. Profesora Escuela Profesional de Psicología, UAP. Perú. Correo electrónico: lourdespantigoso@hotmail.com

${ }^{6}$ Licenciado en Educación. Estudios de doctorado en Ciencias de la Educación. Profesor Escuela Profesional de Enfermería, UAP. Perú. Correo electrónico: serafinelangelbueno2@,hotmail.com

${ }^{7}$ Ing. de Sistemas. Especialista en estadística. Doctorando en Ciencias de la Educación. Profesor Escuela Profesional de Ing. Civil, UAP. Perú. Correo electrónico: joselinop@hotmail.com 


\title{
Perception of risks and drug use in university nursing students, Arequipa, Peru ${ }^{1}$
}

\author{
Fidel Urday-Concha ${ }^{2}$, Caridad Gonzáles-Vera ${ }^{3}$, Lorenzo Jesús Peñalva Suca ${ }^{4}$, Esther Lourdes Pantigoso-
}

Bustamante $^{5}$, Serafin Humberto Cruz Sánchez ${ }^{6}$, Lino Pinto-Oppe ${ }^{7}$

Institution: Alas Peruanas University (UAP) and National University of San Agustín (UNSA)

\begin{abstract}
The objective of the present study was to have a first approximation about the prevalence of life, information, perceptions, and risk behaviors regarding the consumption of licit, illicit and medical drugs, according to sex, in nursing students. A quantitative, exploratory descriptive and cross-sectional, census-type study was performed. A standardized questionnaire of 241 items was applied and previously applied to 86 students of the third year of nursing at a private university in Arequipa Metropolitana. There were high prevalence of life for the consumption of licit substances, $84.4 \%$ for alcoholic beverages and $62.8 \%$ for tobacco, and for any illegal drug (31.4\%), among which marijuana $(17.1 \%)$, and cocaine $(4.6 \%)$, with differences in consumption in favor of men, whereas in medical drugs the most commonly used substance is tranquilizers $(19.8 \%)$, with differences in consumption favoring women. At the university, more than $93 \%$ of students received information to prevent drug use, and $34.8 \%$ reported having had sexual intercourse between one and several times under the influence of alcohol and illicit drugs. It is concluded that the results show the magnitude of the social problems of the consumption of licit, illicit and medical drugs among university students; In addition, there is a need to develop strategies for the promotion of health and prevention of psychoactive substances in the nursing career to reduce and prevent the prevalence of consumption among future professionals promoting healthy lifestyles, to ensure a better quality of life in the population.
\end{abstract}

Keywords: illicit-drugs; student-nursing; perception-of-risks; prevalence.

DOI: $10.15517 /$ revenf.v0i36.33416

${ }^{1}$ Date of receipt: May 23, 2018

Date of acceptance: October 4, 2018

${ }^{2}$ Sociologist. PhD in Sociology and PhD in Biomedical Sciences. Professor of Postgraduate School, Alas Peruanas University (UAP) and National University of San Agustín (UNSA), Arequipa, Peru. E-mail: fidelurdayconcha@hotmail.com

${ }^{3}$ Nurse. Master's Studies in Nursing Sciences. Main Professor to D.E., UNSA. Peru. E-mail: caridadgonzalesvera@hotmail.com

${ }^{4}$ Sociologist. Doctor in Higher Education. Professor Professional School of Nursing, UAP. Peru. E-mail: jesusleo55@,hotmail.com

${ }^{5}$ Psychologist. Drug dependence specialist. Professor Professional School of Psychology, UAP. Peru. E-mail:

lourdespantigoso@hotmail.com

${ }^{6}$ Bachelor of Education. PhD studies in Education Sciences. Professor Professional School of Nursing, UAP. Peru. E-mail: serafinelangelbueno2@hotmail.com

${ }^{7}$ Systems Engineer. Statistician PhD student in Education Sciences. Professor Professional School of Civil Engineering, UAP. Peru. Email: joselinop@hotmail.com 


\section{Percepção de riscos e uso de drogas em estudantes de enfermagem universitária, Arequipa, Peru ${ }^{1}$}

Fidel Urday-Concha ${ }^{2}$, Caridad Gonzáles-Vera ${ }^{3}$, Lorenzo Jesús Peñalva Suca ${ }^{4}$, Esther Lourdes PantigosoBustamante $^{5}$, Serafin Humberto Cruz Sánchez ${ }^{6}$, Lino Pinto-Oppe ${ }^{7}$

Instituição: Universidade Alas Peruanas (UAP) e Universidade Nacional de San Agustín (UNSA)

\section{RESUMO}

O objetivo do presente estudo foi ter uma primeira aproximação sobre a prevalência de vida, informação, percepções e comportamentos de risco em relação ao consumo de drogas lícitas, ilícitas e médicas, segundo o sexo, em estudantes de enfermagem. Foi realizado um estudo quantitativo, descritivo exploratório e de corte transversal, tipo censo. Um questionário padronizado de 241 itens foi aplicado previamente a 86 estudantes do terceiro ano de enfermagem de uma universidade privada de Arequipa Metropolitana. Elevada prevalência de vida para o consumo de substâncias lícitas em bebidas alcoólicas $84,4 \%$ e $62,8 \%$ foi encontrada em rapé, e qualquer droga $31,4 \%$, entre os quais marijuana $(17,1 \%)$ e cocaína $(4,6 \%)$, com diferenças de consumo em favor dos homens, enquanto em medicamentos mais vulgarmente utilizados substância corresponde a tranquilizantes $(19,8 \%)$, com diferenças de consumo em favor das mulheres. Além disso, na faculdade mais de $93 \%$ dos alunos receberam informações para prevenir o uso de drogas e 34,8\% relataram ter tido relações sexuais uma a várias vezes sob a influência de álcool e drogas ilícitas. Conclui-se que os resultados mostram a magnitude da problemática social do consumo de drogas lícitas, ilícitas e médicas entre os universitários; Além disso, há necessidade de desenvolver estratégias de promoção da saúde e prevenção do uso de substâncias psicoativas na carreira de enfermagem para reduzir e prevenir que a prevalência do consumo aumente nos futuros profissionais promovendo estilos de vida saudáveis, a fim de garantir uma melhor qualidade de vida na população.

Palavras chave: drogas-ilícitas; estudante-enfermagem; percepção-de-riscos; prevalência.

DOI: $10.15517 /$ revenf.v0i36.33416

${ }^{1}$ Data de recepção: 23 de maio de 2018

Data de aceitação: 4 de outubro de 2018

2 Sociólogo. Doutor em Sociologia e Doutor em Ciências Biomédicas. Professor da Escola de Pós-Graduação da Universidade Alas Peruanas (UAP) e da Universidade Nacional de San Agustín (UNSA), Arequipa, Peru. Correio eletrônico:

fidelurdayconcha@hotmail.com

${ }^{3}$ Enfermeira. Mestrado em Ciências da Enfermagem. Professor Principal de D.E., UNSA. Peru. Correio eletrônico:

caridadgonzalesvera@hotmail.com

${ }^{4}$ Sociólogo. Doutor em Ensino Superior. Professora Escola Profissional de Enfermagem, UAP. Peru. Correio eletrônico: jesusleo55@hotmail.com

${ }^{5}$ Psicólogo. Especialista em dependência de drogas. Professora da Escola Profissional de Psicologia da UAP. Peru. Correio eletrônico: lourdespantigoso@hotmail.com

${ }^{6}$ Bacharel em Educação. Doutorado em Ciências da Educação. Professora Escola Profissional de Enfermagem, UAP. Peru. Correio eletrônico: serafinelangelbueno2@hotmail.com

${ }^{7}$ Engenheiro de Sistemas. Estatístico Doutoranda em Ciências da Educação. Professor da Escola Profissional de Engenharia Civil, UAP. Peru. Correio eletrônico: joselinop@,hotmail.com 


\section{INTRODUCCIÓN}

El consumo de drogas o sustancias psicoactivas ${ }^{1-3}$ y sus consecuencias socio-epidemiológicas para las personas, sus familias y la sociedad representan un grave problema de salud pública a nivel mundial y, particularmente, para Latinoamérica y Perú $^{4-8} 2015$; lo cual se refleja en el incremento constante de las estadísticas mundiales del número de consumidores y defunciones ocasionadas.

El World Drug Report de la Oficina contra la Droga y el Delito de la Organización de las Naciones Unidas [UNODC/ONU] del $2017^{9}$, señala que en el 2015 unos 250 millones de personas en el mundo (alrededor del 5\% de la población con edades entre los 15-64 años) consumieron alguna droga ilícita por lo menos una vez, y según el número de consumidores en los 12 meses anteriores al 2015, la marihuana fue la sustancia psicoactiva más consumida en el mundo (183 millones), seguida de las anfetaminas y estimulantes de venta con receta (37 millones), opioides (35 millones), éxtasis (22 millones) y cocaína (17 millones). A lo anterior, cabe agregar que el consumo de drogas ilícitas sigue causando daños irreparables en la salud y la pérdida de vidas valiosas: al respecto, en el año 2014 ocasionó un total aproximado de 207,400 muertes ${ }^{10}$; no obstante, dado que a nivel mundial casi 12 millones de personas consumen drogas por inyección, mueren más consumidores de drogas a causa de la hepatitis C (222,000 muertes) que por VIH/SIDA (60,000 muertes), según reporta la UNODC/ONU en el 2017.

Las consecuencias sanitarias y sociales del uso de sustancias ilícitas, por demás preocupantes, son superadas ampliamente por el de las drogas sociales (alcohol y tabaco) en el mundo, sin dejar de lado que, como revela la Organización Mundial de la Salud [OMS], el consumo excesivo y nocivo de alcohol "provoca efectos sanitarios y sociales perjudiciales para el bebedor" ${ }^{\prime 1}$, su familia y la sociedad. Además, es un factor causal en más de 200 enfermedades y trastornos mentales, y ocasiona la muerte de más de 3,3 millones de personas cada año, lo que representa un $5,9 \%$ de todas las defunciones mundiales ${ }^{12}$.

Respecto del consumo de tabaco, la OMS y la Organización Panamericana de la Salud [OPS] reportan que en el 2013 había aproximadamente 1,200 millones de fumadores, de lo que 145 millones residen en América; es decir, el $12 \%$ del total mundial. En cuanto al consumo de tabaco y la exposición al humo de tabaco de segunda mano, causa aproximadamente seis millones de defunciones cada año de las que alrededor de un millón corresponden a la región de las Américas ${ }^{13}$.

Respecto de los estudiantes universitarios en las Américas cada vez se observa una mayor propensión a involucrarse en el consumo de drogas, hecho que origina el incremento de las prevalencias de consumo alguna vez en la vida, así como en el último año y último mes.

En relación con lo anterior, una investigación con estudiantes de ciencias médicas de la Universidad Nacional Autónoma de Nicaragua revela un $52,6 \%$ de prevalencia de vida de consumo de alcohol, seguido por tabaco $(25,4 \%)$ y consumo de medicamentos con $48,7 \%{ }^{14}$. Otro estudio en una universidad tradicional chilena observa que las drogas lícitas no solo han sido las más probadas, sino casi la totalidad han consumido alcohol (96,4\%), seguido por el tabaco (71\%), marihuana (44,1\%) y tranquilizantes con un $13,8 \%{ }^{15}$, mientras que el $51,9 \%$ de la muestra del alumnado de medicina de la Universidad de Manizales, Colombia, aseveró haber consumido algún tipo de estimulantes para mejorar el rendimiento académico ${ }^{16}$. 
Luego, en una investigación específica con estudiantes de enfermería de la Universidad de Costa Rica, en cuanto al consumo de drogas lícitas e ilícitas señala que el 58\% declaró haberlas usado y el 39,1\% manifestó que no le llamaba la atención el utilizar drogas ${ }^{17}$. En cambio, en otra más reciente con estudiantes de enfermería de dos universidades privadas del área Metropolitana de Monterey, Nuevo León, México, respecto a la prevalencia de vida, se observa que el $83,1 \%$ ha consumido alcohol, y el $56 \%$ tabaco mientras que, en relación con la prevalencia de consumo en el último año, el 53\% afirmó haber consumido alcohol y el 41,6\% tabaco ${ }^{18}$.

En concreto, los resultados de las diversas investigaciones en las Américas en estudiantes universitarios revelan, en primer lugar, el incremento paulatino de la prevalencia del consumo de drogas alguna vez en la vida, entre las que se destaca alcohol, tabaco, marihuana y estimulantes ${ }^{14,17,19}$. En segundo, los motivos por los que se usa drogas lícitas e ilícitas es por satisfacer la curiosidad, por diversión, placer o pasarla bien; para acompañar a su pareja o amigos, para relacionarse con otras personas, fácil acceso, pertenecer a un grupo de pares, etc. ${ }^{1720-23}$. Por último, señala que las edades de inicio del consumo de drogas legales, ilegales y médicas son cada vez más tempranas ${ }^{18,20,23}$.

En Perú, el consumo de drogas es un problema de salud pública, por su magnitud cada vez más creciente, la tendencia de uso hacia edades de inicio más tempranas, el incremento del poli-consumo y mezclas de sustancias psicoactivas, etc. Particularmente, una importante proporción de estudiantes universitarios cada vez tienen una mayor predisposición a involucrarse y experimentar alguna vez en la vida con el consumo de drogas y, en algunos casos, de convertirse en consumidores ocasionales y habituales, incluso, de llegar a una situación de abuso y dependencia fisiológica o física, psicológica y social ${ }^{8,24-26}$.

En relación con Perú, el proyecto Programa Anti-Drogas Ilícitas en la Comunidad Andina [PRADICAN] 2013 destaca que el $87,1 \%$ y el $59,9 \%$ de los estudiantes universitarios declaró haber consumido alcohol y tabaco respectivamente alguna vez en la vida, indicadores que descienden al $71,7 \%$ y $36,2 \%$ durante el último año (consumo reciente), mientas que, excluyendo al alcohol, tabaco y fármacos, un 5,7\% de los estudiantes declaró haber usado alguna droga ilícita en el último año, con diferencias significativas por sexo $(7,7 \%$ de los hombres versus $3,9 \%$ de las mujeres) ${ }^{27}$.

Otro estudio sobre las opiniones, percepciones, actitudes y comportamientos de riesgo asociados al consumo de drogas en estudiantes de psicología de dos universidades particulares de Lima Metropolitana muestra diferencias estadísticamente significativas en la prevalencia de vida del consumo de las siguientes drogas en función del sexo: marihuana, sustancias cocaínicas y de síntesis $(\mathrm{P}<0.05)$. Respecto de la percepción de riesgo de drogas ilícitas, la droga más peligrosa es la pasta básica de cocaína combinada con marihuana (91\%) y en cuanto a los comportamientos de riesgo más de un tercio reportó haber tenido relaciones sexuales bajo los efectos de las drogas ilícitas o alcohol ${ }^{28}$.

Por último, una investigación de Lorenzo, Cajaleón y Gutiérrez (2014), en estudiantes de Nutrición de una universidad de Lima, Perú, indica que la prevalencia de vida para el consumo de alcohol fue de $78,8 \%$ y para el tabaco $42,8 \%$; respecto de la edad de inicio, el grupo etario más grande de los estudiantes $(50,7 \%)$ inició el consumo de bebidas alcohólicas en el transcurso de los 15 a 17 años, con una edad promedio de inicio de $17 \pm$ 
2.1; mientras que en cuanto al consumo de tabaco, la edad de inicio del grupo etario más grande $(53,3 \%)$ se encuentra entre los 15-17 años, cuya edad promedio de inicio es de $17 \pm 2^{28}$.

En consecuencia, motivados por el progresivo incremento en el consumo de drogas, según los antecedentes mencionados, y como en Arequipa no se han realizado investigaciones específicas que exploren el problema de las drogas en estudiantes universitarios, el objetivo del presente estudio es lograr una primera aproximación referencial sobre la prevalencia de vida, información recibida, percepciones, opiniones y comportamientos de riesgo respecto al consumo de drogas lícitas, ilícitas y médicas, según sexo, en estudiantes de enfermería de una universidad particular de Arequipa Metropolitana.

Dado que conocer las magnitudes de las variables mencionadas es una condición sine qua non para iniciar el análisis, la explicación y la formulación de alternativas de solución en un campo profesional que tiene como misión la educación y la promoción de la salud, así como el cuidado de la vida humana, para contribuir a coadyuvar la adopción de estilos de vida saludables, alcanzar el bienestar social y una mayor calidad de vida en la población en general, son imprescindibles; por lo tanto, el propósito es obtener información de interés para diseñar un estudio más representativo y con un diseño explicativo en torno a los factores de riesgo y protección del consumo de drogas en estudiantes universitarios de Arequipa.

\section{MATERIALES Y MÉTODO}

La presente investigación se realizó con estudiantes universitarios matriculados en el tercer año, ciclos V y VI, segundo semestre de 2014, de la Escuela de Enfermería de una universidad privada de Arequipa Metropolitana. La población del estudio fue seleccionada de manera intencional, alumnado matriculado en el tercer año de enfermería (125) y, por tanto, el método de muestreo es no probabilístico. Del total de la población teórica, el instrumento se aplicó a 94 estudiantes y la tasa de éxito fue de 91,6\%. El criterio de inclusión fue haber completado el cuestionario hasta el módulo de alcohol, mientras que el principal criterio de eliminación fue todos los casos en que los participantes respondieran "no" a la pregunta "por la presente, declaro que entrego esta información de manera voluntaria". Para realizar el análisis estadístico de los datos y la interpretación de los resultados, la muestra total del estudio fue de 86 estudiantes: 79 (91,9\%), mujeres y 7 (8,1\%) varones.

El estudio corresponde a un diseño no experimental, de corte transversal y tipo descriptivo exploratorio (Hernández, Fernández y Baptista, 2003). El enfoque para el estudio de las variables fue cuantitativo y focalizado en dos ciclos de estudios de la Licenciatura en Enfermería.

En función del objetivo, para recolectar la información se aplicó un cuestionario auto diligenciado, estandarizado, previamente evaluado y validado en la población universitaria de los países andinos, entre la Comisión Interamericana para el Control del Abuso de Drogas de la Organización de los Estados Americanos [CICAD/OEA] y los profesionales del proyecto "Programa Antidrogas Ilícitas en la Comunidad Andina" (PRADICAN, 2013), el cual contiene seis módulos y 241 preguntas con alternativas de respuesta mixta, cerrada y abierta. 
El presente estudio solo exploró el análisis e interpretación de las siguientes variables: sociodemográficas: sexo, edad y ocupación; consumo de drogas lícitas, ilícitas y médicas: frecuencia de consumo, edad de inicio y percepción de riesgo de consumo; opinión: acceso y oferta de sustancias ilícitas; información recibida: frecuencia y valoración de la información; comportamiento de los participantes: conductas de riesgo relacionadas con el consumo de sustancias legales e ilegales.

La aplicación del cuestionario estructurado fue realizada en los salones de clase seleccionados en días y horas previamente pactadas, previa coordinación con la Dirección de la Escuela de Enfermería y los docentes de las asignaturas seleccionadas. En vista de que completar los cuestionarios tomó alrededor de 35 minutos, se evitó aplicarlos en la última hora de clase y en la semana previa a la evaluación de las asignaturas.

Previo a recolectar la información, el equipo de investigación explicó a los estudiantes los objetivos y beneficios de la investigación. En cada salón de clases se resaltó la participación voluntaria del alumnado, el carácter confidencial y reserva en el manejo de su información, para que garanticen la calidad de los datos y asuman el compromiso de involucrarse en el estudio. Luego, se entregó a cada participante un cuestionario dentro de un sobre manila: utilizando el mismo procedimiento, lo devolvieron sin ningún tipo de identificación personal.

Culminado el trabajo de campo, el primer paso fue revisar los cuestionarios individualmente antes de ser digitalizados, para corregir las probables incongruencias, y eliminar aquellos que no lograron satisfacer los criterios de inclusión y exclusión, con la finalidad de garantizar la consistencia y calidad de la información obtenida. En segundo lugar, se procedió a codificar las variables y dimensiones de estudio con sus respectivas alternativas de respuesta e ingresar la información obtenida en una base de datos.

La información fue procesada y analizada en el programa Microsoft Office Excel 2007; para el análisis estadístico se describió las variables cuantitativas y cualitativas: las primeras a través de frecuencias y medidas de tendencia central, y las segundas a través del análisis descriptivo y proposiciones de tipo teórico.

\section{Consideraciones éticas}

El estudio tuvo aprobación y autorización escrita del Comité de Ética de la Escuela Profesional de Enfermería, siempre y cuando se incluya los principios éticos básicos en relación con la investigación en sujetos humanos: respeto por las personas, beneficencia y justicia.

\section{RESULTADOS}

\section{Caracterización social de los participantes}

En relación con las características sociodemográficas de la población de estudio, respecto de la variable sexo, $91,9 \%$ corresponde a mujeres y $8,1 \%$, a varones, mientras que, en cuanto a la edad, los grupos de mayor predominio fueron de 20 a 22 años $(38,4 \%)$ y de 23 a 25 años $(22,1 \%)$. El promedio de edad de la población fue 
de 23 años y la desviación estándar de 3,6 años. En lo concerniente a la ocupación, solo el 21\% reportó que estudia y trabaja, mientras que una mayoría significativa (79\%) solo estudia.

En la tabla 1 se muestra la prevalencia de vida y la edad de inicio de consumo de drogas: en torno al consumo de drogas lícitas, la información evidenció que el $88,4 \%(\mathrm{n}=76)$ del estudiantado de enfermería asegura haber consumido alguna bebida alcohólica al menos una vez en la vida y el $62,8 \%(n=54)$ reconoció haber fumado cigarrillos, por lo que la edad de inicio promedio de consumo para las mujeres es de 15,1 años; no obstante, es oportuno resaltar que más del $37 \%$ de los estudiantes no ha probado el tabaco.

En cuanto al consumo de drogas ilícitas, la marihuana ha sido la más consumida $(17,4 \% ; n=15)$ : particularmente el $57 \%$ del total de los hombres la han consumido alguna vez en la vida, seguido por el consumo de cocaína e inhalantes con un 4,6\%, en ambos casos. Al analizar el consumo de estas drogas por sexo, los hombres que probaron cocaína e inhalantes representan el $28,6 \%(\mathrm{n}=2)$ y $42,9 \%(\mathrm{n}=3)$ respectivamente, mientras que las mujeres solo alcanzan el 2,5\% (n=2) y 1,3\% $(\mathrm{n}=1)$. En relación con las drogas de uso médico, el 19,8\% $(\mathrm{n}=17)$ expresó haber consumido tranquilizantes y el 16,3\% $(n=14)$ estimulantes al menos una vez en la vida .

Tabla 1. Universidad privada de Arequipa Metropolitana. Distribución de Edad de inicio y prevalencia de vida de estudiantes, según sexo, de consumo de drogas legales, ilegales y médicas. (Frecuencia absoluta: fi; Frecuencia absoluta acumulada: $F i=79$ mujeres; $F i=7$ hombres; Frecuencia relativa: $n_{i}$; Frecuencia relativa acumulada: $N i$ )

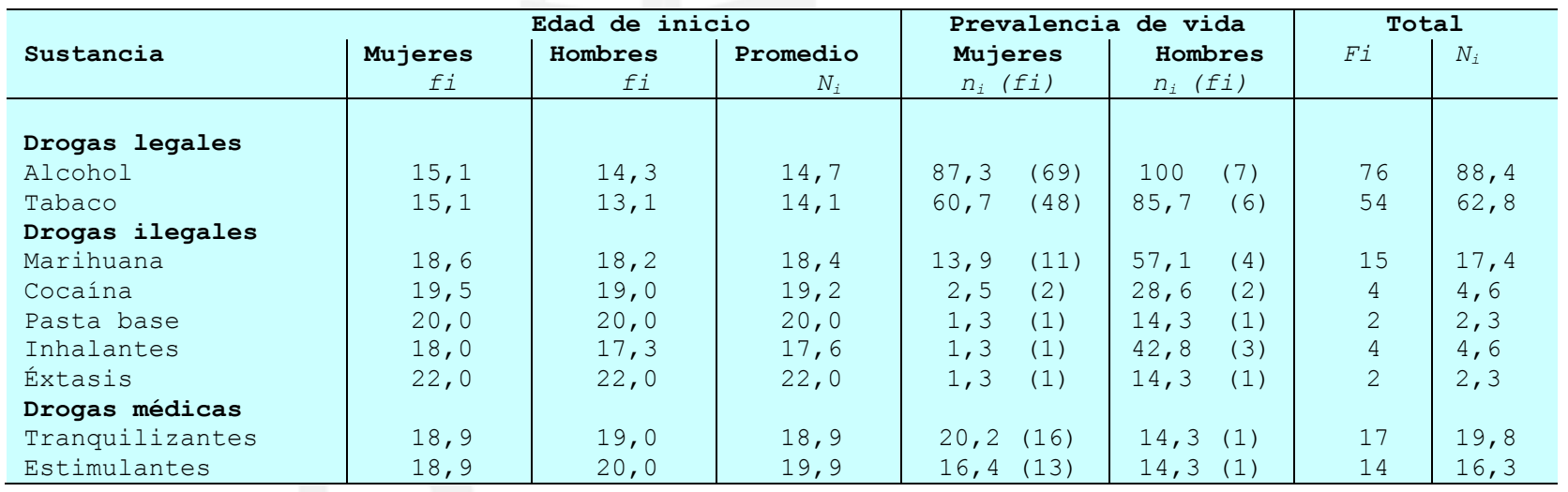

Fuente: cuestionario auto-administrado.

En cuanto a la información sobre el tema de las sustancias psicoactivas, el 39,5\% ( $\mathrm{n}=34)$ de los estudiantes manifestó haber recibido información en la enseñanza secundaria. En términos de frecuencia, el 41,2\% (n=14) la recibió pocas veces y más de la mitad de los participantes $(52,9 \% ; n=18)$ la calificó como buena (tabla 2). 
Tabla 2. Universidad privada de Arequipa Metropolitana. Distribución de la información recibida sobre el tema de drogas en educación secundaria y universitaria. (Frecuencias absolutas $\left[f_{i}\right]$ y relativas $\left[n_{i}\right]$ )

\begin{tabular}{l|c|c|c|c}
\hline & \multicolumn{2}{|c|}{ Secundaria } & \multicolumn{2}{c}{ Universidad } \\
Frecuencia y valoración & $\boldsymbol{f}_{\boldsymbol{i}}$ & $\boldsymbol{n}_{\boldsymbol{i}}$ & $\boldsymbol{f}_{\boldsymbol{i}}$ & $\boldsymbol{n}_{\boldsymbol{i}}$ \\
\hline Recibió información & & & & \\
Sí & 34 & 39,5 & 82 & 93,3 \\
No & 52 & 60,5 & 4 & 4,7 \\
Frecuencia de información & & & & \\
Una sola vez & 12 & 35,3 & 11 & 13,4 \\
Pocas veces & 14 & 41,2 & 42 & 51,2 \\
Varias o muchas veces & 2 & 5,9 & 27 & 32,9 \\
No recuerda & 6 & 17,6 & 2 & 2,4 \\
Valoración de la información & & & & \\
Muy buena & 6 & 17,6 & 25 & 30,5 \\
Buena & 18 & 52,9 & 48 & 58,5 \\
Regular y deficiente* & 5 & 14,7 & 7 & 8,3 \\
No recuerdo & 5 & 14,7 & 2 & 2,4 \\
\hline
\end{tabular}

Fuente: cuestionario auto-administrado

* Mala y muy mala para la información recibida en secundaria

En la universidad, una gran mayoría de los estudiantes de enfermería $(93,3 \% \mathrm{n}=82)$ recibió información para prevenir el uso de drogas, con claras diferencias entre mujeres y hombres: 96,2\% y 85,7\% respectivamente. Respecto de la frecuencia de la información recibida, más de la mitad $(51,2 \% \mathrm{n}=42)$ la recibió pocas veces y el $89 \%$ de los participantes la valoró como buena y muy buena.

En cuanto a la percepción de riesgo sobre el consumo frecuente de alcohol, el 79,1\% de los estudiantes de enfermería percibió dicha conducta como de gran riesgo o muy peligrosa: esta percepción, según el sexo, es bastante mayor respecto de las mujeres $(78,5 \%)$ en comparación con los hombres $(71,4 \%)$. A su vez, casi el $77 \%$ de los participantes reveló como gran riesgo el uso frecuente de tabaco y sólo una proporción inferior al 10\% está de acuerdo que el uso frecuente de alcohol y tabaco tienen un riesgo leve para la salud.

En torno a la percepción social de riesgo de las drogas ilegales, casi la totalidad del alumnado (94,2\%) afirmó que la droga más peligrosa o de gran riesgo es el consumo frecuente de pasta básica de cocaína (pbc), seguida por los usos frecuentes de cocaína (93\%), éxtasis $(90,7 \%)$ y marihuana $(87,2 \%)$; no obstante, preocupa que el 9,3\% piensa que el uso frecuente de marihuana tiene un riesgo leve para la salud, seguido por el uso frecuente de tranquilizantes con un $12,8 \%$. Se observó, además, según el sexo, las mujeres tienen una marcada mayor percepción de gran riesgo respecto del uso frecuente de pasta base de cocaína, cocaína y éxtasis, etc., en comparación con los hombres.

Tras consultar a los estudiantes sobre la facilidad o dificultad percibida para el acceso a sustancias psicótropas ilícitas, la tabla 3 evidenció que el 19,8\% $(\mathrm{n}=17)$ de los jóvenes considera que podría conseguir fácilmente marihuana, seguido por un $8,1 \%(\mathrm{n}=7)$ de cocaína. Empero, también se puede observar que en promedio dos tercios de los encuestados $(66,6 \%)$ perciben no saber si es fácil o difícil acceder a drogas, mientras que, según sexo, el indicador promedio es mucho mayor entre las mujeres (70,5\%) en comparación con los hombres (22,9\%). 
Tabla 3. Universidad privada de Arequipa Metropolitana. Distribución de las opiniones respecto a dificultades y oferta para acceder a drogas ilegales. (Frecuencias absolutas $\left[f_{i}\right]$ y relativas $\left[n_{i}\right]$ )

\begin{tabular}{|c|c|c|c|c|c|c|c|c|}
\hline \multirow{3}{*}{ Drogas } & \multicolumn{4}{|c|}{ Dificultades } & \multicolumn{4}{|c|}{ Oferta } \\
\hline & \multicolumn{2}{|c|}{ Fácil } & \multicolumn{2}{|c|}{$\begin{array}{c}\text { No sé si es fácil } \\
\text { o difícil }\end{array}$} & \multicolumn{2}{|c|}{$\begin{array}{c}\text { En el último } \\
\text { año }\end{array}$} & \multicolumn{2}{|c|}{$\begin{array}{l}\text { Nunca me } \\
\text { ofrecieron }\end{array}$} \\
\hline & $f_{i}$ & $n_{i}$ & $f_{i}$ & $n_{i}$ & $f_{i}$ & $n_{i}$ & $f_{i}$ & $n_{i}$ \\
\hline Marihuana & 17 & 19,8 & 53 & 61,6 & 15 & 17,1 & 48 & 55,8 \\
\hline Cocaína & 7 & 8,1 & 54 & 62,8 & 9 & 10,5 & 47 & 54,6 \\
\hline Pasta básica & 6 & 7,0 & 56 & 65,1 & 4 & 4,6 & 54 & 62,8 \\
\hline Heroína & 0 & 0,0 & 61 & 70,9 & 0 & 0,0 & 62 & 72,1 \\
\hline Éxtasis & 4 & 4,6 & 58 & 67,4 & 3 & 3,5 & 59 & 68,6 \\
\hline Anfetaminas* & 0 & 0,0 & 58 & 67,4 & 0 & 0,0 & 61 & 70,9 \\
\hline Metanfetaminas** & 1 & 1,2 & 57 & 66,3 & 1 & 1,2 & 63 & 73,2 \\
\hline Ketamina & 0 & 0,0 & 58 & 67,4 & 0 & 0,0 & 61 & 70,9 \\
\hline LSD & 0 & 0,0 & 58 & 67,8 & 0 & 0,0 & 61 & 70,9 \\
\hline Poppers & 0 & 0,0 & 59 & 68,6 & 0 & 0,0 & 62 & 72,1 \\
\hline
\end{tabular}

Fuente: cuestionario auto-administrado.

* Pastis, speed o aceleradores ** Meth, hielo, cristales, etc.

La tabla 4 reportó que bajo los efectos del alcohol, el 13,9\% $(n=12)$ del estudiantado del tercer año de enfermería solo una vez ha tenido relaciones sexuales y un $11.6 \%(\mathrm{n}=10)$ manifestó haber tenido relaciones sexuales varias veces y, según el sexo, en los dos casos se evidenció un mayor comportamiento de riesgo en los varones en comparación con las mujeres. No obstante, el 71,9\% $(\mathrm{n}=61)$ de los encuestados reveló que bajo los efectos del alcohol nunca han tenido relaciones sexuales, con una marcada mayor frecuencia de las mujeres $(75,9 \%)$ en comparación con los varones $(14,3 \%)$.

Tabla 4. Universidad privada de Arequipa Metropolitana. Distribución de los comportamientos de riesgo respecto al consumo de algunas drogas legales e ilegales. (Frecuencias absolutas $\left[f_{i}\right]$ y relativas $\left[n_{i}\right]$ )

\begin{tabular}{|c|c|c|c|c|c|c|}
\hline \multirow[t]{2}{*}{ Riesgos por consumo de drogas } & \multicolumn{2}{|c|}{ Sólo una vez } & \multicolumn{2}{|c|}{ Varias veces } & \multicolumn{2}{|c|}{ Nunca } \\
\hline & $f_{i}$ & $\boldsymbol{n}_{i}$ & $f_{i}$ & $n_{i}$ & $f_{i}$ & $\boldsymbol{n}_{i}$ \\
\hline $\begin{array}{l}\text { ¿Ha tenido relaciones sexuales bajo } \\
\text { los efectos delalcohol? }\end{array}$ & 12 & 13,9 & 10 & 11,6 & 61 & 70,9 \\
\hline $\begin{array}{l}\text { ¿Ha tenido relaciones sexuales bajo } \\
\text { el efecto de drogas ilícitas? } \\
\text { ¿Ha conducido un vehículo motorizado }\end{array}$ & 6 & 7,0 & 2 & 2,3 & 72 & 83,7 \\
\hline $\begin{array}{l}\text { en los últimos } 12 \text { meses después de } \\
\text { beber alcohol? }\end{array}$ & 7 & 8,1 & 4 & 4,6 & 69 & 80,2 \\
\hline $\begin{array}{l}\text { ¿Ha viajado en los últimos } 12 \text { meses } \\
\text { en algún vehículo motorizado } \\
\text { conducido por alguien bajo los } \\
\text { efectos del alcohol? }\end{array}$ & 11 & 12,8 & 23 & 26,7 & 52 & 60,5 \\
\hline
\end{tabular}

Fuente: cuestionario auto-administrado.

En cuanto a los comportamientos de riesgo respecto del consumo de drogas ilícitas el 7\% ( $\mathrm{n}=6$ ) declaró haber tenido relaciones sexuales solo una vez bajo los efectos del consumo de marihuana, cocaína, éxtasis u otras drogas. Sin embargo, es pertinente resaltar que el $83,7 \%(n=72)$ del estudiantado manifestó que nunca ha tenido relaciones sexuales bajo los efectos del consumo de drogas ilícitas. 


\section{DISCUSIÓN}

La presente investigación reportó que la población del tercer año de la Escuela de Enfermería era una población joven, predominantemente de sexo femenino y la gran mayoría del alumnado sólo se dedicaba a estudiar. Estos resultados sociodemográficos han sido corroborados por otros autores que tuvieron como unidades de análisis estudiantes universitarios de enfermería ${ }^{17-18,20,30-32}$.

Las sustancias psicoactivas de mayor consumo reportadas por los jóvenes universitarios son las sociales (alcohol y tabaco) y su problemática es creciente. Este estudio encontró que el alcohol es la droga legal más consumida, con una prevalencia de vida del 88,4\% y, según diferencias por sexo, el uso es mayor en hombres que en mujeres. Tal resultado es similar a lo reportado por otras investigaciones realizadas en el Perú en muestras aleatorias de estudiantes universitarios de psicología ${ }^{24}$ y en la población universitaria en general ${ }^{27}$, así como semejante al resultado que revela el estudio efectuado por el Centro de Información y Educación para la Prevención del Abuso de Drogas [CEDRO, 2011a] en la población peruana entre los 12 y 64 años ${ }^{33}$.

En contraste, el resultado es inferior según indica un estudio en jóvenes universitarios de Cali, Colombia ${ }^{19}$, y muy superior al consumo de alcohol entre estudiantes de ciencias médicas de una universidad de Nicaragua ${ }^{14}$, mientras que es casi similar al consumo de alcohol que reporta una investigación en estudiantes de enfermería de dos universidades privadas de Nuevo León, México ${ }^{18}$. Estos estudios respecto a la prevalencia de vida del consumo de alcohol por sexo, también evidencian un mayor consumo entre los hombres que en mujeres.

Dado lo anterior, esta investigación evidenció que el consumo de bebidas alcohólicas es la droga legal más consumida por los jóvenes universitarios. En segundo lugar, también representa el principal problema de salud pública del país, por sus graves efectos en el organismo humano, la desestructuración familiar, el ausentismo laboral y exposición a accidentes de tránsito y conductas temerarias ${ }^{34}$.

Una situación semejante se observó con el tabaco, cuya prevalencia de vida de consumo fue de $62,8 \%$ entre el alumnado encuestado de enfermería, hallazgo contrario a los resultados encontrados en jóvenes universitarios de nuestro país ya que, por un lado, una investigación en estudiantes de psicología en Lima Metropolitana reporta una prevalencia de vida de consumo de tabaco relativamente más baja, 59,9\% ${ }^{24} \mathrm{y}$, por otro lado, el PRADICAN ${ }^{27}$ observa un indicador más elevado, 70,8\%, empero el indicador mencionado arriba es ligeramente superior en comparación con la prevalencia de vida del consumo de tabaco (56\%) de una población de estudiantes de enfermería de dos universidades privadas de Nuevo León, México ${ }^{18}$.

Al respecto, es importante aclarar que las diferencias entre los indicadores descritos, podrían ser resultado del empleo de diferentes métodos de análisis y contraste, debido a que en este estudio se utilizó una muestra intencional para recolectar la información y, en los otros, se evidencia muestras representativas. No obstante, a pesar de las diferencias descritas es más importante resaltar que el consumo de tabaco tiene graves consecuencias sociales y de salud en la población: es el principal factor de riesgo de las enfermedades crónicas no trasmisibles y "la primera causa de mortalidad prevenible en el mundo"13.

En relación con el consumo de las denominadas sustancias duras, en total, esta investigación reportó que un $31,2 \%$ de los estudiantes ha probado algún tipo de las drogas ilícitas en estudio alguna vez en la vida, indicador 
superior a las tasas de consumo total de drogas ilícitas reportadas para las poblaciones universitarias de los países $\operatorname{andinos}^{35}$ y del Perú ${ }^{27}$.

La droga ilegal más consumida alguna vez en la vida por los jóvenes universitarios de enfermería es la marihuana $(17,4 \%)$ y, según sexo, existen claras diferencias en su uso entre hombres $(57,1 \%)$ y mujeres $(13,9 \%)$, resultados que corroboran los hallazgos de algunos informes mundiales en los que el cannabis sigue siendo la droga ilícita de mayor consumo a nivel mundial ${ }^{7,35-37}$. Al resaltar estudios latinoamericanos en poblaciones universitarias, la tasa de consumo de marihuana arriba en mención observó una cercana similitud con el resultado que indica una muestra de estudiantes de psicología de dos universidades de Lima Metropolitana $-18,4 \%{ }^{24}$ - y es mayor en comparación, primero, con el de una muestra universitaria de Perú $-11,6 \%{ }^{27}-\mathrm{y}$, segundo, en estudiantes de la salud y ciencias sociales $-11,9 \%$ - de la Universidad de Santander, Colombia ${ }^{32}$, pero es inferior respecto de la prevalencia de vida reportada en una muestra de estudiantes de una universidad de Medellín $-36,3 \%^{21}$ - y de una universidad tradicional chilena $-44,1 \%{ }^{15}$.

Las tasas de prevalencia de vida del consumo de cocaína, inhalantes, pasta base y éxtasis que se reveló en el presente estudio son mucho mayores a las reportadas por el PRADICAN ${ }^{27}$ para estudiantes universitarios y para la población urbana peruana ${ }^{32}$. Es importante resaltar que a diferencia de estas investigaciones nacionales, algunos estudios latinoamericanos destacan indicadores mayores para el consumo de cocaína $-10,5 \%{ }^{21}$ - y $-5,3 \%{ }^{15}$-, así como para el uso del éxtasis y otras anfetaminas $-9,4 \%{ }^{21}{ }^{-}$. Es probable que las tasas de consumo de estas drogas ilícitas sean menores en los estudiantes universitarios de enfermería, a diferencia de la marihuana, en virtud a la información, cursos o talleres recibidos en la universidad, así como de una mayor percepción de riesgo en cuanto a su consumo y sus efectos en la salud y vida de la población universitaria.

En relación al consumo de drogas médicas, sustancias de venta lícita y cotidianamente adquiridas en el mercado con o sin prescripción médica, en esta investigación también se reportó indicadores altos de prevalencia de vida para el consumo de estimulantes $(16,3 \%)$ y tranquilizantes $(19,8 \%)$ y, según sexo, a diferencia del consumo de las drogas ilícitas, se observó que las mujeres tienen una prevalencia de vida de consumo de drogas médicas más alta respecto a los hombres, incluso, el resultado del consumo de tranquilizantes que se identificó es superior a los indicadores reportados por los tres estudios arriba mencionados, $10,5 \%{ }^{27}, 12,2 \%{ }^{24}$ y $13,8 \%{ }^{15}$ y por una investigación en estudiantes de la Universidad de Sonora (5,3\%), México ${ }^{39}$. En el caso del consumo de estimulantes, se identificó que es significativamente menor $(16,3 \%)$ respecto de la información revelada $(51,9 \%)$ por una investigación en estudiantes de medicina de la Universidad de Manizales, Colombia ${ }^{16}$.

En cuanto a la información recibida en la universidad sobre el tema de las drogas y cuya frecuencia se reportó en el presente estudio $(93,3 \%)$, no solo es muy superior a la observada $(56,2 \%)$ por una investigación en estudiantes de psicología de dos universidades privadas de Lima Metropolitana ${ }^{24}$, sino también es 3,2 veces mayor que la revelada por el proyecto PRADICAN ${ }^{27}$ para la población universitaria del Perú. En torno a lo anterior, es oportuno resaltar que, a diferencia de esta investigación en la cual se aplicó el instrumento solo a estudiantes del tercer año de enfermería, en los dos estudios comentados líneas arriba se aplicó el instrumento a una muestra aleatoria de estudiantes matriculados del primero al quinto año: el primer estudio la aplicó solo al alumnado de la carrera de psicología y, el segundo, a estudiantes de todas las carreras de las áreas de ciencias y letras de 10 universidades públicas y privadas de Perú, situaciones que podrían haber influido en las diferencias de las tasas en cuanto a la información recibida. 
En cuanto a la percepción de riesgo sobre el consumo de drogas ilícitas, los hallazgos de esta investigación revelaron que en promedio el $92,6 \%$ de los estudiantes de enfermería percibe el uso frecuente de pasta básica de cocaína, cocaína y éxtasis, como de gran riesgo o peligrosidad para la salud y, según sexo, se observó una mayor percepción de gran riesgo en las mujeres que en los hombres en el uso frecuente de drogas lícitas e ilícitas; por lo tanto, los resultados podrían evidenciar indicadores epidemiológicos ligeramente superiores a los encontrados por otras investigaciones en muestras de estudiantes universitarios de los países andinos ${ }^{35}$, Perú (PRADICAN, 2013) y dos universidades de Lima Metropolitana ${ }^{24}$.

En el caso de la percepción de riesgo sobre el consumo frecuente (último mes) de drogas farmacológicas, a pesar de los altos índices en cuanto a la frecuencia de la información recibida, pocas o varias veces, y la valoración de la información tipificada como buena o muy buena, para prevenir el consumo de drogas en la universidad, llama la atención que casi el 13\% y más del 9\% de los encuestados opina que el uso frecuente de tranquilizantes y estimulantes genera un riesgo leve para la salud. Tales resultados podrían estar favorecidos por su amplia disponibilidad, venta libre, precios bajos en farmacias locales y la cultura de la automedicación que existe en nuestro medio ${ }^{16,27,31}$, así como el uso de tranquilizantes sea para moderar la ansiedad o para poder dormir ${ }^{26}$, mientras que los estimulantes son usados por motivos académicos, la alta exigencia y carga académica ${ }^{30}$, particularmente para mejorar el rendimiento académico en estudiantes de medicina ${ }^{15}$ y enfermería, según testimonios reportados.

Un indicador importante que refleja la problemática social del consumo de drogas es la práctica de conductas o comportamientos de riesgo de los jóvenes universitarios: en torno a lo anterior, este estudio encontró que el 34,8\% de los estudiantes de enfermería declaró haber tenido relaciones sexuales solo una vez y varias veces bajo los efectos del consumo de alcohol y otras drogas ilegales. Tal frecuencia es semejante a la reportada (34,4\%) en una muestra de estudiantes universitarios de Lima Metropolitana ${ }^{24}$, incluso, reveló también que el 37,2\% de los participantes han viajado en los últimos 12 meses solo una vez y varias veces en algún vehículo motorizado conducido por una persona bajo los efectos del alcohol.

En consonancia con lo anterior, es probable que la evidencia estadística del estudio demostrara dos hechos en cuanto a las conductas de riesgo relacionadas con el consumo de drogas legales e ilegales. En primer lugar, los comportamientos de riesgo más frecuentes son haber viajado en alguna oportunidad como pasajero en un vehículo motorizado conducido por una persona que estaba bajo los efectos del alcohol y haber tenido relaciones sexuales bajo los efectos del alcohol y alguna droga ilícita. En segundo lugar, cuando se indagó por relaciones sexuales, los hombres tienen una marcada mayor exposición a los comportamientos de riesgo en comparación con las mujeres.

\section{CONCLUSIONES}

Los resultados de este estudio indican palmariamente una alta prevalencia de vida del consumo de sustancias psicoactivas lícitas e ilícitas en estudiantes de enfermería, de las que el alcohol es la droga de mayor consumo $(88,4 \%)$, seguido del tabaco $(62,8 \%)$ y de la marihuana $(17,4 \%)$. No obstante, particularmente la prevalencia de vida en cuanto al consumo de alcohol es similar y el tabaco es superior, casi en ocho puntos, en relación con los indicadores mencionados por una investigación previa en una población de estudiantes de enfermería de dos universidades privadas de Monterrey, Nuevo León, México. 
En segundo lugar, respecto de la percepción de riesgo del consumo frecuente de alcohol se evidencia también que casi ocho de cada 10 estudiantes perciben dicha conducta como de gran riesgo o muy peligrosa, mientras que en relación con las drogas ilícitas nueve de cada 10 indican que el consumo frecuente de pasta básica, cocaína y éxtasis son las drogas más peligrosas o de gran riesgo para la salud. Sin embargo, estos resultados son contradictorios con los indicadores epidemiológicos reportados principalmente en lo concerniente a las prevalencias de vida del consumo de drogas lícitas.

Por último, como corolario, se concluye que el profesional de enfermería en el acto de cuidado por la vida y promoción de la salud, debe efectuar un análisis integral de la problemática social del consumo de drogas, participar en forma activa en la prevención de los comportamientos de riesgo, la sensibilización y educación de las poblaciones universitarias a través de la introducción del tema en el currículo de estudios para morigerar, prevenir y plantear soluciones adecuadas al consumo de sustancias psicoactivas como problema de salud pública. tales retos podrían justificar no solo la especialización y la apertura de un nuevo campo laboral, sino una mayor valorización del cuidado de la vida llevada a cabo por la/el enfermera/o ${ }^{40}$, en las instituciones públicas y privadas de salud, la familia y la comunidad.

\section{AGRADECIMIENTOS}

Agradecemos al Director de la Escuela de Postgrado de la Universidad Alas Peruanas, Dr. José Luque, y a la Directora de la Escuela de Posgrado, Coordinadora de Enfermería, Universidad Nacional de San Agustín de Arequipa, Dra. Sonia Velásquez, por darnos la oportunidad de exponer los avances del presente estudio entre los doctorandos de Derecho y Enfermería. Por último, nuestro más sincero agradecimiento al Soc. Lino Benavente Butrón por revisar y releer el presente artículo para asegurar la coherencia que debe existir en materia de diseño, análisis y resultados del estudio.

\section{REFERENCIAS BIBLIOGRÁFICAS}

1. Becoña E. Bases científicas de la prevención de las drogodependencias. Madrid: Ministerio del Interior / Delegación del Gobierno para el Plan Nacional sobre Drogas; 2012.

2. Becoña E, Cortés T. editores. Manual de adicciones para psicólogos especialistas en psicología clínica en formación. Barcelona: SOCIDROGALALCOHOL; 2010.

3. Martín M, Lorenzo P. Conceptos fundamentales en drogodependencias. En: Lorenzo P, Ladero J M, Leza J C, Lizasoain I, coords. Drogodependencias. Farmacología. Patología. Psicología. Legislación. 3a ed. Madrid: Editorial Médica Panamericana; 2009. p.1-25.

4. Centro de Información y Educación para la Prevención del Abuso de Drogas. El problema de las drogas en el Perú. Lima: CEDRO; 2015.

5. Chávez-Hernández AM, Macías-García LF, Páramo-Castillo D, Martínez C, Ojeda D. Consumo de drogas en estudiantes de nivel medio superior de la Universidad de Guanajuato. Acta Universitaria.2005; 15(001): 13-21. 
6. Gobierno de España. Guía sobre drogas. Realidades, mitos, efectos, tipos, riesgos, abuso, consumo, dependencia, drogas. Madrid: Ministerio de Sanidad y Consumo; 2009.

7. United Nations Office on Drugs and Crime. World Drug Report 2012. Disponible en http://www.unodc.org/unodc/secured/wdr/wdr2013/World Drug Report 2013.pdf

8. Zavaleta A, Romero E, Castro de la Mata R. Variables asociadas a la prevalencia de vida de drogas en jóvenes de Lima-Perú. En: Zavaleta A. editor. Factores de riesgo y protección en el consumo de drogas en la juventud. Lima:

CEDRO; 2001: 3-49.

9. Oficina de las Naciones Unidas contra la Droga y el Delito. Resumen, Conclusiones y Consecuencias en Materia de Políticas. Informe Mundial de las Drogas 2017. Disponible en http://www.unodc.org/wdr2017

10. Oficina de las Naciones Unidas contra la Droga y el Delito. Resumen Ejecutivo. Informe Mundial sobre las Drogas 2016. Disponible en https://www.unodc.org/doc/wdr2016/WDR 2016 ExSum spanish.pdf

11. Organización Mundial de la Salud. Consumo de alcohol y salud en el mundo. Disponible en http://blogs.20minutes.es/el-nutricionista-d-la-general/2014/19/informe-oms-2014-consumo de-alcohol-y-salud-en-elmundo/

12. World Health Organization. Global status report in alcohol and health 2014. Disponible en http://apps.who.int/iris/bitstream/10665/112736/1/9789240692763_eng.pdf

13. Organización Panamericana de la Salud y Organización Mundial de la Salud. Informe sobre Control del Tabaco para la Región de las Américas 2013. Washington D.C.: OPS; 2013. Disponible en http://www.paho.org/hq/index.php?option=com_docman\&task=doc view\&gid=23415

14. Castro N, Cortez P, Pereira G, Lobo da Costa M. Uso de drogas entre estudiantes de la Facultad de Ciencias Médicas de la Universidad Nacional Autónoma de Nicaragua, León, Nicaragua. Revista Latino-Am. Enfermagem. 2010; 18 ( $\mathrm{N}^{\circ}$. Spec): 606-12.

15. Sepúlveda MJ, Roa J, Muñoz M. Estudio cuantitativo del consumo de drogas y factores sociodemográficos asociados en estudiantes de una universidad tradicional chilena. Rev. med. Chile. 2011; 139 (7): 856-863.

16. Barón L, Botero-Henao K, Castaño-Castrillón J J, Castillo-Chang K, Díaz-Corrales J, Echeverri-Uribe JS, et al. Prevalencia y factores asociados al consumo de anfetaminas, en estudiantes del Programa de Medicina de la Universidad de Manizales (Colombia), 2010. Rev Fac Med. 2011; 59 (3): 2001-2014.

17. Rojas L, Lobo da Costa M, Pereira G. Caracterización de estudiantes de la carrera de licenciatura de enfermería y sus percepciones sobre el consumo de drogas lícitas e ilícitas. Revista Latino-Americana de Enfermagem. $2010 ; 18\left(\mathrm{~N}^{\circ}\right.$ Spec,): 535-542.

18. Telumbre-Terrero J Y, Esparza-Almanza SE, Alonso Castillo VA, Alonso-Castillo M T. Consumo de alcohol y Tabaco en estudiantes de enfermería. Revenf. 2016; 30: 1-16. Doi: http://dx.doi.org/10.1551/revenf.vOi30.22020- 
19. Cáceres D, Salazar I, Varela M, Tovar J. Consumo de drogas en jóvenes universitarios y su relación de riesgo y protección con los factores psicosociales. Rev. Universitas Psychologica. 2006; 5 (3): 521-534.

20. Leiva V. Características relacionadas con el consumo de drogas lícitas e ilícitas en la población estudiantil de cuarto año de la carrera de Enfermería. Revenf. 2009; 15: 1-14.

21. Tirado AF, Álvarez M, Velásquez JD, Gómez L, Ramírez C, Vargas AR. Prevalencia y factores de riesgo para el consumo y dependencia de drogas en estudiantes de una universidad de Medellín, Colombia, 2009. Revista Facultad Nacional de Salud Pública. 2012; 30 (1): 38-44.

22. Vargas M, Arena CA, Silva da EC. Conocimientos de estudiantes de enfermería sobre alcohol y drogas. Revista Latino-Americana de Enfermagem. 2010; 18 (N. Spec): 529-534.

23. Zapata MA, Segura AM. Consumo de drogas y riesgos asociados en jóvenes escolarizados de la ciudad de Medellín. 2007. Revista Salud Pública de Medellín. 2008; 3 (2): 7-20.

24. Espinoza L. Opiniones, percepciones, actitudes y comportamientos asociados al consumo de drogas en estudiantes universitarios de psicología de Lima Metropolitana: Un enfoque cuantitativo y cualitativo. Revista Peruana de Drogodependencias. Análisis e Investigación. 2011; VII (1): 7-41.

25. García ME, Borasino P, Amaya EM. Conductas sexuales de riesgo post consumo de alcohol y/o drogas ilegales en estudiantes en carreras no médicas de la Universidad Los Ángeles de Chimbote, Centro Académico Sullana, durante diciembre 2008 - 2009. Rev. Peruana de Drogodependencias. 2011; VII (1): 83-96.

26. Rubio G. Aspectos biopsicosociales de las drogodependencias. En: Martínez M, Rubio G, editores. Manual de Drogodependencias para Enfermería. 19-29. Madrid: Ediciones Díaz de Santos, S. A; 2002. p. 19-29.

27. Proyecto Programa Antidrogas Ilícitas en la Comunidad Andina. Estudio Epidemiológico Andino sobre Consumo de Drogas en la Población Universitaria. Informe Perú, 2012. Lima: CAN/UNION EUROPEA / DEVIDA / UNODC; 2013.

28. Lorenzo M, Cajalión B, Gutiérrez E. Prevalencia y factores asociados al consumo de alcohol y tabaco en estudiantes de nutrición de una universidad de Lima-Perú. Rev. Perú. Epidemiol.2012;16(3):01-05.

29. Hernández R, Fernández C, Baptista P. Metodología de la investigación. 3a ed. México: Mc Graw-Hill. 2003.

30. Morales B, Plazas M, Sánchez R, Arena, C. Factores de riesgo y de protección relacionados con el consumo de sustancias psicoactivas en estudiantes de enfermería. Rev. Latino-Am. Enfermagem. 2011; 19 (No.sp,): 673-83.

31. Vargas E, Barrio V, Campos M, Porras D, Quesada K, Leiva V. La salud internacional y el consumo de drogas por parte de la población adolescente de Costa Rica. Revenf. 2010; 17: 1-18.

32. Páez AN, Solano S, Durán M, Mansilla D, Suárez E, Melgarejo P, et al. Prevalencia del consumo de sustancias psicoactivas en estudiantes universitarios de la salud y ciencias sociales. Rev. CUIDARTE. 2012; 3, (1): 334-341.

33. Centro de Información y Educación para la Prevención del Abuso de Drogas. Epidemiología de drogas en la población urbana peruana 2010. Encuesta de Hogares. Monografía de Investigación N 26. Lima: CEDRO,2011a. Disponible en http://www.bvcedro.org.pe/bitstream/123456789/118/1/236-CEDRO.pdf 
34. Centro de Información y Educación para la Prevención del Abuso de Drogas. El Problema de las Drogas en el Perú. 25 años. En la lucha contra las drogas y la mejora de la calidad de vida. Lima: CEDRO, 2011b. Disponible en: https://www.google.com.pe/?gfe rd=cr\&ei=OBnnVZCSNI6w8we9q7XwBA\&gws rd=ssl\#q=CEDRO+\%2B+EL+PRO BLEMA+DE+LAS+DROGAS+EN+EL+PERU+\%2B+25+A $\% \mathrm{C} 3 \% 91 \mathrm{OS}+\mathrm{MAS}+\mathrm{EN}+\mathrm{LA}+\mathrm{LUCHA}+\mathrm{CONTRA}+\mathrm{LAS}+$ DROGAS+Y+MEJORA+DE+LA+CALIDAD+DE+VIDA

35. Proyecto Apoyo a la Comunidad Andina en el Área de Drogas Sintéticas. Estudio Epidemiológico Andino sobre Consumo de Drogas Sintéticas en la Población Universitaria. Informe Comparativo: Bolivia, Colombia, Ecuador y Perú. Lima: CAN/UNION EUROPEA/DEVIDA/OPD; 2009.

36. United Nations Office on Drug and Crime. World Drug Report 2013. Disponible en https://www.unodc.org/unodc/.../wdr2013/World_Drug_Report 2013.pdf

37. Oficina de las Naciones Unidas contra la Droga y el Crimen. Resumen Ejecutivo. Informe Mundial sobre las Drogas 2014. Disponible en http://www.unodc.org/documents/wdr2014/v1403603 spanish.pdf

38. Oficina de las Naciones Unidas contra la Droga y el Delito (UNODC). Informe Mundial sobre las drogas 2015. Resumen Ejecutivo. URL. disponible en: https://www.unodc.urg/documents/wdr2015/WSR15 ExSum S.pdf

39. Olivares AJ, Favela MA, Tirad L, Barragán O, Hernández EA. Consumo de drogas y competencia social en jóvenes universitarios de la Unidad Regional Sur. Rev. EPISTEMUS. 2013; 15: 5-22.

40. Wright M. Liderazgo internacional en Enfermería relacionada con el fenómeno de las drogas: un estudio de caso de la experiencia de colaboración entre la Comisión Interamericana para el Control del Abuso de las Drogas (CICAD) y la Universidad de Alberta-Facultad de Enfermería. Rev. Latino-Am. Enfermagem. 2005; 13 (2): 1-26. 